\title{
Sintomas depressivos e eventos estressores em crianças e adolescentes no contexto de institucionalização
}

\author{
Depressive symptoms and stressful events in children and adolescents in the \\ institutionalized context
}

\begin{abstract}
Josiane Lieberknecht Wathier'1, Débora Dalbosco Dell'Aglio²
${ }^{1}$ Graduada. Mestranda, Programa de Pós-Graduação em Psicologia, Universidade Federal do Rio Grande do Sul (UFRGS), Porto Alegre, RS. ${ }^{2}$ Doutora em Psicologia do Desenvolvimento, UFRGS. Docente, Programa de Pós-Graduação em Psicologia, Instituto de Psicologia, UFRGS.

Este artigo constitui parte da dissertação de mestrado da primeira autora, intitulada "Vivências adversas e depressão: um estudo sobre crianças e adolescentes institucionalizados” (em andamento), desenvolvida no Curso de Pós-Graduação em Psicologia do Desenvolvimento, Universidade Federal do Rio Grande do Sul, Porto Alegre, RS. A pesquisa foi desenvolvida dentro de um projeto maior financiado pelo Edital Pronex do Conselho Nacional de Desenvolvimento Científico e Tecnológico (CNPq) e da Fundação de Amparo à Pesquisa do Estado do Rio Grande do Sul (FAPERGS), intitulado "A instituição, a escola e a rua como contextos ecológicos de promoção de resiliência para jovens em situação de risco".
\end{abstract}

\section{Resumo}

Introdução: O presente estudo verificou a manifestação de sintomas depressivos e a freqüência e o impacto de eventos estressores em crianças e adolescentes institucionalizados e não-institucionalizados.

Método: Participaram 257 jovens de 7 a 16 anos de ambos os sexos, sendo que 130 residiam em abrigos de proteção e 127 moravam com suas famílias na região metropolitana de Porto Alegre. Todos os participantes freqüentavam da $1^{\mathrm{a}}$ à $8^{\mathrm{a}}$ série do ensino fundamental de escolas públicas localizadas em bairros de baixas condições socioeconômicas. Foram aplicados os instrumentos Children's Depression Inventory e o Inventário de Eventos Estressores na Infância e Adolescência.

Resultados: Foram encontradas médias mais altas no Children's Depression Inventory e no Inventário de Eventos Estressores na Infância e Adolescência no grupo institucionalizado, com diferença significativa. Os eventos mais freqüentes foram "ter que obedecer às ordens de seus pais" para o grupo da família e "ser levado para um abrigo" no outro grupo. Os eventos que apresentaram maior impacto foram "morte de um dos pais", "ser estuprado", "ser rejeitado pelos familiares” e "ser tocado sexualmente contra a vontade”. Foram encontradas diferenças entre os sexos e entre os contextos no impacto atribuído aos eventos. Os adolescentes, independentemente dos contextos, apresentaram freqüência de eventos significativamente maior do que as crianças, o que não ocorreu quanto ao impacto.

Conclusão: Os jovens abrigados apresentaram maior exposição a situações potenciais de risco, o que pode estar relacionado à maior manifestação de sintomas depressivos. A avaliação do impacto de um evento estressante, no entanto, não esteve correlacionada com o Children's Depression Inventory em nenhum dos contextos.

Descritores: Criança, adolescente, sintomas depressivos, eventos estressantes.

\begin{abstract}
Introduction: The present study examined the manifestation of depressive symptoms, as well as frequency and impact of stressful events in institutionalized and non-institutionalized children and adolescents.

Method: A total of 257 youths, of both genders, aged 7-16 years were included in this study. Of these, 130 lived in protection youth shelters and 127 lived with their families in the metropolitan area of Porto Alegre. All participants were attending 1st to 8th grades of public elementary schools located in low-income neighborhoods. Children's Depression Inventory and Stressful Events in Childhood and Adolescence Inventory were used.
\end{abstract}


Results: Data pointed out a significant difference, with higher means in the Children's Depression Inventory and Stressful Events in Childhood and Adolescence Inventory for the institutionalized group. The most frequent events for the family group was "to have to obey your parent's rules," and "to be taken to a protection shelter" was most frequent for the other group. The events of greater impact were "death of one parent," "to be raped," "to be rejected by members of the family," and "to be sexually touched against one's will". Considering the participant's gender and context, differences were found in attributed impact. Adolescents, regardless of context, had a higher frequency of significant events than children, which did not occur concerning impact.

Conclusion: Results indicated that institutionalized youths were more exposed to potential risk situations, which could be related to the manifestation of more depressive symptoms. However, assessment of the impact of a stressful event was not correlated with the Children's Depression Inventory in any context.

Keywords: Children, adolescents, depressive symptoms, stressful events.

\section{Introdução}

A depressão em crianças e adolescentes vem sendo bastante investigada, pois afeta diversos campos da vida dos jovens, causando significativos danos psicossociais ${ }^{1,2}$. Destacam-se pesquisas que investigam a depressão em jovens que vivenciaram eventos estressores, como violência, abandono e perdas ${ }^{3-7}$. No entanto, encontram-se poucos estudos específicos com a população de crianças e adolescentes que vivem em abrigos de proteção no Brasil, se comparados aos dos jovens em contexto hospitalar ou escolar, por exemplo.

O termo depressão tem sido usado em diferentes amplitudes, que variam desde a designação de um estado afetivo normal, apresentação de sintomas, até a caracterização de uma síndrome e doença específica ${ }^{8}$. Na abordagem clínica da depressão, estudos têm evidenciado as particularidades da sintomatologia da depressão em crianças e adolescentes ${ }^{2,9,10}$. A depressão na infância e adolescência pode ter início com a perda de interesse pelas atividades que normalmente seriam atrativas, manifestando-se como uma espécie de mau humor constante diante dos jogos, brincadeiras e esportes.

Quanto à investigação da depressão, variados métodos têm sido utilizados, com diferentes critérios diagnósticos, instrumentos e tipo de pontuação estudada. Embora não sejam destinadas ao diagnóstico, escalas de depressão como o Beck Depression Inventory (Cunha ${ }^{11}$ ) e o Children's Depression Inventory (CDI) (Kovacs ${ }^{12}$ ) mostraram que têm capacidade de discriminar grupos já diagnosticados com transtorno depressivo maior, distimia e transtorno depressivo com desordem de ajustamento, entre outros. Os critérios utilizados para esses estudos foram os do Diagnostic and Statistical Manual of Mental Disorders, DSM-III ${ }^{13}$ e DSM-IV ${ }^{14}$ (American Psychiatric Association). Entretanto, é possível estender esses achados aos critérios do DSM-IV-TR ${ }^{15}$, visto que não houve mudanças significativas sobre o construto ou critérios de avaliação dos sintomas.

A prevalência de diagnóstico de depressão varia conforme as características da amostra e do estudo, mas existe um consenso entre os pesquisadores quanto à multiplicidade de fatores sociais, psicológicos e biológicos que envolvem o surgimento da depressão como síndrome ou transtorno ${ }^{9,16-19}$. Entre esses fatores, envolvidos no estudo da depressão, é importante considerarmos os diferentes contextos de desenvolvimento dos jovens e os eventos estressantes vivenciados. Os contextos de desenvolvimento de crianças e adolescentes, seja junto às suas famílias ou em instituições de abrigo, são caracterizados pela presença de fatores de risco e de proteção, podendo se constituir como ambientes desfavoráveis ou promotores de desenvolvimento. Algumas famílias podem experimentar eventos negativos como uma ameaça e não conseguir estabelecer um meio saudável de interações na rede de apoio, ocasionando uma falha em seu papel ${ }^{20,21}$ no desenvolvimento emocional de seus $\operatorname{membros}^{22,23}$.

Eventos estressantes, tais como separação dos pais, família numerosa, história de criminalidade em um dos pais, doença mental dos genitores e baixa renda, são citados como fatores de risco para depressão ${ }^{24-26}$. Sternberg et al. ${ }^{7}$ confirmaram, em um estudo longitudinal, que crianças que sofreram algum tipo de violência intrafamiliar apresentaram mais problemas de comportamento ou sintomas depressivos na adolescência do que as que não foram vítimas. Também Feijó et al. ${ }^{5}$ alertaram que os eventos crônicos ou microeventos diários podem ser mais perturbadores para a saúde mental do que eventos estressores agudos. Dessa forma, situações que envolveram violência, abandono, falta de apoio social, quebra de vínculos, entre outras, poderiam ser potencialmente associadas à depressão, conforme afirmam Dell'Aglio et al ${ }^{4}$. Outros autores apontam, ainda, que vários sintomas psicopatológicos podem estar presentes de forma secundária à exposição a lares violentos e agressivos $^{17,27,28}$. Quando as famílias não conseguem exercer seu papel protetivo, podem ser utilizadas medidas de proteção, como o abrigamento, previsto pelo Estatuto da Criança e do Adolescente (ECA) ${ }^{29,30}$.

Em um estudo de Tolfree, citado por Pinheiro ${ }^{29}$, foi estimado que cerca de 8 milhões das crianças do mundo estavam sob regime de acolhimento residencial, sendo que a maioria delas foi abrigada por motivos de violência 
e desintegração familiar, além de condições econômicas e sociais adversas. No Brasil, Silva ${ }^{31}$ destacou, em 2003, que havia 20 mil crianças e adolescentes vivendo em 589 abrigos que recebiam recursos do Governo Federal, sendo que mais da metade dos jovens pesquisados foi institucionalizada por motivos de pobreza. Entre as referidas instituições, 68,3\% eram não-governamentais. Com base nesses estudos, é possível verificar que as adversidades, crônicas ou agudas, assim como o contexto onde a criança vive e suas características individuais, são algumas variáveis que podem interferir no seu desenvolvimento. Este estudo buscou identificar a presença de sintomas depressivos e de eventos estressores entre crianças e adolescentes que moravam em abrigos de proteção em comparação com os que viviam com suas famílias.

\section{Método}

\section{Participantes}

Participaram 257 jovens de 7 a 16 anos (média - M = 11,30; desvio padrão - DP = 2,10), de ambos os sexos, sendo que 130 residiam em sete instituições de proteção governamentais e duas não-governamentais, 127 moravam com suas famílias na região metropolitana de Porto Alegre e todos freqüentavam da $1^{\mathrm{a}}$ à $8^{\mathrm{a}}$ série do ensino fundamental de escolas públicas.

A partir de listagens de abrigos fornecidas pela Fundação de Proteção Especial do Rio Grande do Sul e pela Fundação de Assistência Social e Cidadania órgãos do governo estadual e municipal, respectivamente -, foram convidados a participar, jovens que se encaixavam na referida faixa etária e não apresentavam dificuldades que impossibilitassem a compreensão do conteúdo dos instrumentos. Esses abrigos estão inseridos na comunidade, na qual essas crianças e adolescentes utilizam recursos de escola, centros de lazer, praças e também a rede municipal de saúde. Para compor a amostra dos participantes que moravam com a família (grupo-controle), foram selecionadas, por conveniência, duas escolas públicas localizadas em bairros com índice de vulnerabilidade social inferiores a 0,65 , sendo que, quanto mais próximo de zero, mais vulnerável socialmente é o bairro ${ }^{32}$.

\section{Instrumentos}

Foram utilizados dois instrumentos padronizados, conforme especificado a seguir.

\section{Children's Depression Inventory}

Instrumento elaborado por Kovacs ${ }^{12}$ e adaptado do Beck Depression Inventory para adultos. O objetivo do CDI é detectar a presença e a severidade do transtorno depressivo, identificando alterações afetivas em crianças e adolescentes. É composto por 27 itens, com três opções de resposta, dentre as quais o participante deve escolher a que melhor descreve o seu estado no período atual. O CDI já foi adaptado para uso no Brasil (Inventário de Depressão Infantil), em João Pessoa, por Gouveia et al. ${ }^{33}$, e vem demonstrando características psicométricas adequadas. Estudos com amostras brasileiras apresentam consistência interna variando de 0,79 a $0,92^{4,19,34}$. Kovacs $^{12}$ recomenda que o ponto de corte para indicar provável psicopatologia seja acima de um desvio padrão e meio da média de escore da amostra.

Inventário de Eventos Estressores na Infância e Adolescência (IEEIA)

Adaptado do Inventário de Eventos Estressores na Adolescência (IEEA) (Kristensen et al. ${ }^{35}$ ), este inventário contém 60 itens, que identificam quais os eventos negativos que já aconteceram com o participante e qual a sua percepção quanto ao impacto de cada um desses eventos. Para cada item, o participante deve indicar se o evento ocorreu (sim ou não) e, a partir disso, pontuar em uma escala do tipo Likert de cinco pontos o impacto atribuído a cada evento de fato experienciado. O instrumento demonstrou, em seu estudo original, uma elevada consistência interna (alpha de Cronbach $=0,92$ ), mas não apresenta estudos de normatização.

\section{Procedimentos e considerações éticas}

Esse estudo caracterizou-se por ser de risco mínimo aos participantes e foi aprovado pelo Comitê de Ética da UFRGS, tendo seguido os preceitos éticos que regem a realização de pesquisas com seres humanos (Ministério da Saúde ${ }^{36}$, Conselho Federal de Psicologia ${ }^{37}$ ). Foi obtido o Termo de Consentimento Livre e Esclarecido dos pais ou responsáveis das crianças que moravam com sua família, assim como o Termo de Concordância da Instituição, na figura dos diretores dos abrigos que mantêm a guarda formal dos jovens que ali vivem. Além disso, os adolescentes maiores de 12 anos, de ambos os contextos, também assinaram um Termo de Consentimento Livre e Esclarecido. Para os menores de 12 anos, nos dois contextos, foi assegurada a voluntariedade de sua participação e obteve-se um assentimento verbal. Os instrumentos foram aplicados nas próprias escolas e abrigos, em horários combinados com antecedência, por uma equipe de pesquisa composta por psicólogos e acadêmicos de psicologia previamente treinados. Em situações em que a equipe de coleta observou participantes com desconforto emocional, os casos 
foram discutidos com os psicólogos das instituições para os devidos encaminhamentos.

\section{Análise estatística}

Os dados dos instrumentos foram digitados em uma tabela do programa SPSS, versão 14.0, a partir da qual se realizou análises descritivas e inferenciais. Foram verificadas as consistências internas (alpha de Cronbach) dos instrumentos. Também foram realizados os testes $t$ de Student, qui-quadrado e correlação de Pearson para verificar diferenças e associações entre as variáveis investigadas.

\section{Resultados}

\section{Dados sociodemográficos da amostra}

De uma amostra inicial de 297 jovens que participaram do presente estudo, foram retirados aqueles que não responderam integralmente a todos os itens, tanto do IEEIA como do CDI. Após esse ajuste no banco de dados, foram considerados para levantamento os 257 jovens que haviam respondido aos dois instrumentos de forma completa.

Participaram 66 meninas (50,8\%) e 64 meninos $(49,2 \%)$ institucionalizados $(\mathrm{M}=11,29$ anos; $\mathrm{DP}=$
$2,04)$ e 58 meninas ( $45,7 \%)$ e 69 meninos $(54,3 \%)$ que moravam com a família ( $\mathrm{M}=11,30$ anos; $\mathrm{DP}=2,17)$. Os 130 participantes do contexto dos abrigos de proteção apresentaram um tempo de institucionalização que variou de 1 a 150 meses $(\mathrm{M}=$ 33,49 meses; $\mathrm{DP}=33,38$ ).

\section{Depressão}

O CDI apresentou elevada consistência interna nesse estudo (alpha de Cronbach $=0,86$ ). A média geral da amostra (n = 257) na pontuação do CDI foi de 11,41 $(\mathrm{DP}=8,02)$. Foram realizadas análises considerando as variáveis grupo, sexo e faixa etária (crianças de 7 a 11 anos e adolescentes de 12 a 16 anos). Foram encontradas diferenças significativas quanto ao sexo somente quando separados por grupo, sendo que as meninas institucionalizadas tiveram escores mais altos do que as que moram com a família. Da mesma forma, os dados apontaram uma diferença significativa em relação ao escore de depressão entre os dois grupos por faixa etária, sendo que a média das crianças e adolescentes institucionalizados foi superior à do outro grupo. Não houve, entretanto, diferença significativa entre as médias do CDI por sexo, nem por faixa etária dentro de cada contexto. A Tabela 1 apresenta as médias, desvios padrão e valores do teste $t$ de Student do CDI por grupo, sexo e faixa etária.

Tabela 1 - Média e desvio padrão do Children's Depression Inventory por grupo, sexo e faixa etária

\begin{tabular}{|c|c|c|c|c|c|}
\hline Variável & $\mathbf{n}$ & $\mathbf{M}$ & DP & $t$ & $\mathbf{p}$ \\
\hline \multicolumn{6}{|l|}{ Grupo } \\
\hline Instituição & 130 & 13,26 & 8,53 & $-3,85$ & $<0,01$ \\
\hline Família & 127 & 9,52 & 7,01 & & \\
\hline \multicolumn{6}{|l|}{ Sexo } \\
\hline \multicolumn{6}{|l|}{ Feminino } \\
\hline Instituição & 66 & 14,41 & 9,51 & $-3,30$ & $<0,01$ \\
\hline Família & 58 & 9,45 & 7,16 & & \\
\hline \multicolumn{6}{|l|}{ Masculino } \\
\hline Instituição & 64 & 12,08 & 7,27 & $-2,03$ & $<0,04$ \\
\hline Família & 69 & 9,58 & 6,92 & & \\
\hline \multicolumn{6}{|l|}{ Faixa etária (anos) } \\
\hline \multicolumn{6}{|l|}{ Crianças (7-11) } \\
\hline Instituição & 72 & 13,43 & 9,19 & $-2,85$ & $<0,01$ \\
\hline Família & 64 & 9,28 & 7,58 & & \\
\hline \multicolumn{6}{|c|}{ Adolescentes (12-17) } \\
\hline Instituição & 58 & 13,05 & 7,71 & $-2,56$ & $<0,02$ \\
\hline Família & 63 & 9,76 & 6,44 & & \\
\hline
\end{tabular}

M = média; DP = desvio padrão. 
Kovacs ${ }^{12}$ sugere que um desvio padrão e meio seja utilizado para obter um ponto de corte utilizando o CDI e considerou apropriado um ponto de corte de 19 pontos para o CDI (27 itens) aplicado em sua amostra, assim como Bahls ${ }^{38}$. No presente estudo, considerando como ponto de corte um desvio padrão e meio acima da média (24 pontos), observou-se que 25 casos da amostra total (9,72\%) poderiam ter um provável diagnóstico de depressão. Considerando cada contexto, foram observados seis participantes do grupo da família com escore maior ou igual a 24 pontos $(4,72 \%)$ e 19 do grupo da instituição (14,61\%). Um teste qui-quadrado $\left(\chi^{2}=\right.$ 6,$07 ; \mathrm{gl}=1 ; \mathrm{p}=0,014$ ) revelou associação significativa entre o provável diagnóstico de depressão e o grupo da instituição. Tomando-se como ponto de corte aquele utilizado por Kovacs ${ }^{12}$ e Bahls ${ }^{38}$, de 19 pontos, encontramos 44 casos $(17,1 \%)$ na amostra total, sendo 15 (11,8\%) no grupo da família e 29 (22,3\%) no grupo da instituição; também foi encontrada associação significativa $\left(\chi^{2}=4,27 ; \mathrm{gl}=1 ; \mathrm{p}=0,039\right)$ entre o provável diagnóstico de depressão no CDI e o contexto.

\section{Eventos estressores}

Para descrever os dados do IEEIA, foram realizadas análises dos escores totais de freqüência e impacto da amostra, bem como das diferenças por grupo, sexo e faixa etária. A consistência interna (alpha de Cronbach) da escala em relação à freqüência de eventos foi de 0,88, e em relação ao impacto foi de 0,92.

Em relação à freqüência de eventos, foi encontrada diferença significativa entre as médias dos grupos, sendo que os jovens institucionalizados apresentaram um número maior de eventos do que os que moravam com a família. Em relação à faixa etária, as crianças, assim como os adolescentes, das instituições apresentaram maior número de eventos do que os das famílias. Foi encontrada diferença entre os sexos quanto ao número de eventos vivenciados somente dentro dos respectivos grupos, ou seja, as meninas da instituição tiveram mais eventos que as da família. Isso se repete em relação aos meninos, conforme a Tabela 2.

Tabela 2 - Médias e desvios padrão da freqüência do Inventário de Eventos Estressores na Infância e Adolescência por grupo, sexo e faixa etária

\begin{tabular}{|c|c|c|c|c|c|}
\hline Variável & $\mathbf{n}$ & $\mathbf{M}$ & DP & $t$ & $\mathbf{p}$ \\
\hline \multicolumn{6}{|l|}{ Grupo } \\
\hline Instituição & 130 & 26,77 & 8,39 & $-6,96$ & $<0,0$ \\
\hline Família & 127 & 19,06 & 9,36 & & \\
\hline \multicolumn{6}{|l|}{ Sexo } \\
\hline \multicolumn{6}{|l|}{ Feminino } \\
\hline Instituição & 66 & 26,89 & 7,90 & $-4,97$ & $<0,0$ \\
\hline Família & 58 & 19,21 & 9,30 & & \\
\hline \multicolumn{6}{|l|}{ Masculino } \\
\hline Instituição & 64 & 26,64 & 8,93 & $-4,82$ & $<0,0$ \\
\hline Família & 69 & 18,93 & 9,47 & & \\
\hline \multicolumn{6}{|l|}{ Faixa etária (anos) } \\
\hline \multicolumn{6}{|l|}{ Crianças (7-11) } \\
\hline Instituição & 72 & 25,33 & 8,32 & $-6,26$ & $<0,0$ \\
\hline Família & 64 & 16,69 & 7,71 & & \\
\hline \multicolumn{6}{|c|}{ Adolescentes (12-17) } \\
\hline Instituição & 58 & 28,55 & 8,21 & $-4,17$ & $=0,01$ \\
\hline Família & 63 & 21,46 & 10,28 & & \\
\hline
\end{tabular}

$\mathrm{M}$ = média; $\mathrm{DP}$ = desvio padrão.

Comparando crianças e adolescentes dentro do contexto da instituição, também foi encontrada diferença significativa $(t=-2,21 ; \mathrm{gl}=114,97 ; \mathrm{p}<0,03)$, sendo que a média dos jovens com mais de 12 anos foi mais alta do que a das crianças nesse contexto. Resultado semelhante ocorreu entre os participantes do contexto da família $(t=-2,95 ; \mathrm{gl}=114,97 ; \mathrm{p}<0,05)$, sendo que os adolescentes que moravam com sua família obtiveram média significativamente maior do que as crianças desse contexto. 
Os cinco eventos que tiveram maior freqüência entre os institucionalizados em relação ao outro grupo foram: "ser levado para algum abrigo" (100\%), "ter que viver em abrigo" (98\%), "ter que obedecer às ordens dos pais” (89,4\%), “mudar de colégio” (81,8\%) e “discutir com amigos" (83,3\%). No grupo dos participantes que moravam com a família, os eventos mais freqüentes foram: "ter que obedecer às ordens dos pais” (89,7\%), “morte de outros familiares” (84,5\%), "discutir com amigos" (72,4\%), "tirar notas baixas na escola” (67,2\%) e “ter brigas com irmãos” (70,7\%).
Quanto aos eventos que obtiveram maior impacto, considerando a escala Likert de 1 (nada estressante) a 5 (muitíssimo estressante), foi encontrada diferença significativa do impacto atribuído a alguns eventos específicos para cada grupo, sendo que, na maioria desses eventos, a média maior foi encontrada no grupo da família, conforme pode ser visto na Tabela 3 . Os eventos significativamente mais impactantes para o sexo feminino e para o sexo masculino, por contexto, estão especificados na Tabela 4.

Tabela 3 - Médias e desvios padrão do impacto dos eventos* do Inventário de Eventos Estressores na Infância e Adolescência por grupo

\begin{tabular}{lcccc}
\hline $\begin{array}{l}\text { Grupo } \\
\text { Eventos }\end{array}$ & Instituição M (DP) & Família M (DP) & $\boldsymbol{t}$ & p \\
\hline Família ter problemas com a polícia & $3,55(1,44)$ & $2,96(1,27)$ & $-2,23$ & $<0,03$ \\
Um dos pais ter filhos com outros parceiros & $2,95(1,65)$ & $1,92(1,22)$ & $-4,50$ & $<0,01$ \\
Não ter amigos & $2,95(1,25)$ & $3,57(1,47)$ & 2,27 & $<0,03$ \\
Envolver-se em brigas com agressão física & $3,40(1,41)$ & $3,93(1,37)$ & 2,17 & $<0,04$ \\
Ser expulso da sala de aula pela professora & $2,51(1,53)$ & $3,41(1,24)$ & 2,65 & $<0,01$ \\
\hline
\end{tabular}

$\mathrm{M}=$ média; $\mathrm{DP}$ = desvio padrão.

* Eventos com diferença estatística entre os grupos.

Tabela 4 - Médias e desvios padrão do impacto dos eventos* do Inventário de Eventos Estressores na Infância e Adolescência por grupo em cada sexo

\begin{tabular}{llcccc}
\hline & Eventos & Instituição M (DP) & Família M (DP) & \multicolumn{1}{c}{$\boldsymbol{t}$} & p \\
\hline \multirow{2}{*}{ Meninas } & Família ter problema com a polícia & $3,68(1,47)$ & $2,85(1,35)$ & $-2,03$ & $<0,05$ \\
& Um dos pais ter filhos c/ outros parceiros & $2,93(1,59)$ & $1,96(1,06)$ & $-3,04$ & $<0,01$ \\
& Trabalhar para ajudar a família & $3,20(1,63)$ & $3,09(1,47)$ & $-2,51$ & $<0,02$ \\
\multirow{2}{*}{ Meninos } & Um dos pais ter filhos c/ outros parceiros & $3,02(1,40)$ & $1,94(1,37)$ & $-2,91<0,01$ \\
& Não ter amigos & $2,77(1,51)$ & $3,77(1,41)$ & 2,27 & $<0,03$ \\
& Ir para o Conselho Tutelar & $2,63(1,42)$ & $3,90(1,20)$ & 2,58 & $<0,02$ \\
& Ter mau relacionamento com colegas & $2,37(1,38)$ & $3,56(1,19)$ & 3,06 & $<0,01$ \\
& Envolver-se em brigas & $2,91(1,38)$ & $3,85(1,43)$ & 2,65 & $<0,02$ \\
\hline
\end{tabular}

$\mathrm{M}$ = média; $\mathrm{DP}$ = desvio padrão.

* Eventos com diferença estatística entre os grupos.

Com relação à diferença por faixa etária no impacto, foi encontrada média significativamente maior entre os adolescentes institucionalizados em relação ao outro grupo somente no evento "um dos pais ter filhos com outros parceiros" $(t=2,70 ; \mathrm{gl}=16,93 ; \mathrm{p}<0,01)$, sendo que a média dos jovens institucionalizados $(\mathrm{M}=2,83$;
$\mathrm{DP}=1,65)$ foi mais alta do que entre os que moram com a família $(M=1,60 ; D P=1,07)$. Esse mesmo evento apareceu com média significativamente $(t=-2,23$; $\mathrm{gl}=$ $70 ; \mathrm{p}<0,03)$ mais alta entre as crianças abrigadas $(\mathrm{M}=$ 2,30; DP =1,61), em comparação com as não-abrigadas $(\mathrm{M}=2,30 ; \mathrm{DP}=1,32)$. 


\section{Correlações}

Foi encontrada uma correlação significativa $(r=$ 0,37; p < 0,01) entre o resultado do CDI e o número de eventos estressores, demonstrando que, quanto maior o número de eventos estressores, maior o número de sintomas depressivos. Também foi encontrada correlação significativa entre a idade e o número de eventos ( $r=0,27 ; p<0,01)$. Não foi encontrada correlação significativa entre o impacto dos eventos e CDI por contexto.

\section{Discussão}

Este estudo permitiu verificar os sintomas depressivos e a quantidade de eventos estressores e seu impacto em crianças e adolescentes de dois diferentes contextos de desenvolvimento. No entanto, destaca-se que foi avaliada a presença de sintomas depressivos, mas não no sentido de diagnóstico nosológico. A presença de sintomas depressivos, num grau acima da média da amostra, pode sugerir uma possível síndrome depressiva ou outras categorias nosológicas que compartilham características e sintomas semelhantes, sendo, nesse caso, aconselhável a investigação clínica mais aprofundada para providenciar diagnóstico e tratamento ${ }^{11}$.

Em relação aos pontos de corte utilizados para identificar os jovens com sintomatologia de depressão em cada grupo, é importante destacar que, considerando um desvio padrão e meio acima da média (24 pontos ou mais), o percentual encontrado para a amostra de institucionalizados foi de $14,71 \%$, e considerando o ponto de corte 19, foi de 22,3\%. O estudo de Dell'Aglio et al. ${ }^{3}$ utilizou o escore 29 como ponto de corte (critério de dois desvios padrão acima da média) e apontou um percentual de $6 \%$, em sua amostra, com provável diagnóstico de depressão. Por sua vez, Reppold ${ }^{39}$ utilizou o valor limite de 28 pontos, considerando dois desvios padrão acima da média de uma amostra de adolescentes não-clínicos, e constatou a prevalência de humor depressivo em 5,8\% dos participantes. Cabe ressaltar que a falta de uma norma brasileira consolidada e a aplicação em diferentes amostras relativizam diferenças de escores e passam a ser de difícil comparação. De qualquer forma, os percentuais de provável depressão encontrados na amostra de participantes institucionalizados são altos, embora, para fins de diagnóstico de depressão como transtorno, a avaliação clínica ainda seja indispensável.

Em relação ao sexo, este estudo não apontou diferença significativa entre meninos e meninas quanto aos sintomas depressivos em cada contexto. Nas crianças, essa diferença não tem sido evidenciada, como mostram os estudos de Kovacs ${ }^{40}$, Kim et al. ${ }^{6}$ e Gouveia et al. ${ }^{33}$, assim como em Barbosa et al. ${ }^{41,42}$. Em contrapartida, autores como Dell'Aglio et al. ${ }^{3}$, Cole et al. ${ }^{43}$, além de Reppold ${ }^{39}$, afirmaram ter encontrado diferença significativa entre os sexos utilizando o CDI. Alguns autores enfatizaram que, em amostras de jovens na adolescência inicial, essa diferença de sexo é mais evidente (Batista et al. ${ }^{16}$, Reppold ${ }^{39}$ ), apontando escores mais altos entre as meninas. A partir dos 14 anos, as mulheres apresentam um risco de duas a três vezes maior de desenvolver uma alteração afetiva do que os homens (Reppold ${ }^{39}$ ). O fato de não se ter evidenciado diferença entre os sexos no presente estudo permite inferir que esse instrumento pode medir sintomas depressivos independentemente do sexo, o que demonstraria um de seus aspectos de alta sensibilidade para detectar jovens potencialmente depressivos, como também concluíram Barbosa et al. ${ }^{41}$.

Com relação ao contexto de desenvolvimento dos participantes, foram observados, no presente estudo, maiores escores no CDI entre os jovens que vivem em abrigos de proteção. Em seu estudo utilizando o CDI, Dell'Aglio et al. ${ }^{4}$ também encontraram escores mais elevados entre meninas institucionalizadas do sul do Brasil do que em meninas que moravam com suas famílias. Argumentaram que a família que consegue exercer um papel protetivo ajuda a mediar o impacto dos estressores na vida das adolescentes. Além disso, o resultado encontrado pode estar ligado à quebra de vínculos familiares e, posteriormente, à falta de apoio familiar na mediação do impacto dos eventos estressores na vida dos adolescentes institucionalizados. Outro estudo que investigou a manifestação de sintomas depressivos, a partir do CDI, nos contextos rurais e urbanos, não encontrou diferença significativa entre esses locais de moradia ${ }^{41}$.

Alguns autores demonstraram que crianças e adolescentes que vivem no contexto da instituição têm maior chance de apresentar transtornos psiquiátricos do que aqueles vivendo com suas famílias ${ }^{44,45}$. O argumento de $\mathrm{Abreu}^{44}$ é de que as vidas das crianças e adolescentes abrigados foram marcadas por muitos eventos adversos, o que poderia explicar a associação entre institucionalização e transtornos psiquiátricos. A institucionalização, no estudo dessa pesquisadora, mostrou-se associada a transtornos psiquiátricos, independentemente da presença de outros fatores de risco. Segundo a autora, o primeiro melhor modelo associativo para transtornos psiquiátricos incluiu viver em instituição, ter mãe desaparecida ou falecida, ter desempenho escolar prejudicado e apresentar-se em más condições de higiene. Também Pinheiro ${ }^{29}$, assim como Carvalho $^{46}$, apontou que aspectos como alta rotatividade de funcionários e falta de atividades planejadas e apoio afetivo, por exemplo, podem trazer 
prejuízos aos jovens que se desenvolvem em instituições.

Por outro lado, mudanças significativas no ambiente e na qualidade de atendimento das instituições começam a ser percebidas, conforme Siqueira et al. ${ }^{47}$, sendo que os abrigos têm se mostrado mais adequados ao atendimento e proteção de crianças e adolescentes, de acordo com as especificações do ECA ${ }^{30}$. Assim, a forma como a instituição se organiza para cumprir com as estipulações desse Estatuto pode constituir um fator de risco ou de proteção ao desenvolvimento dos jovens.

Dell'Aglio $^{34}$ investigou os eventos de vida de crianças e adolescentes institucionalizados e nãoinstitucionalizados, através de amostras emparelhadas, também no sul do Brasil. A análise quantitativa dos eventos de vida positivos e negativos não revelou diferença significativa quanto ao número médio de eventos relatados pelos dois grupos. No entanto, observou um número maior de eventos negativos do que de eventos positivos, indicando baixa qualidade de vida em ambos os grupos. Entre os eventos de vida negativos, foram verificados como mais freqüentes aqueles referentes a desentendimentos com pares, doenças e violência.

No presente estudo, os eventos mais freqüentes nos dois contextos, como "ter que obedecer às ordens de seus pais”, “discutir com amigos”, "morte de outros familiares" e "rodar de ano na escola" são semelhantes aos encontrados por Kristensen et al..$^{35}$ Esses achados também vêm ao encontro daqueles encontrados por Dell’Aglio ${ }^{34}$, considerando-se a importância da relação entre os pares e as dificuldades vivenciadas na escola. No entanto, os eventos mais freqüentes não foram os de maior impacto. Os eventos de maior impacto, independentemente do contexto ("morte de um dos pais", "ser estuprado", "ser rejeitado pelos familiares" e "ser tocado sexualmente contra a vontade"), estavam direta ou indiretamente relacionados à família, demonstrando, dessa forma, a importância da mesma.

No entanto, vivenciar uma situação estressante pode ou não vir a ser chamada de situação de risco. Rutter $^{48}$ destaca que os mecanismos de risco devem ser analisados enquanto processos, não sendo uma variável isolada em si mesma. As crianças e adolescentes que moram com suas famílias e que participaram deste estudo também admitiram ter sofrido diversos tipos de violência, embora em menor número, podendo-se concluir que também estão expostas a situações de vulnerabilidade social e pessoal. Por outro lado, observou-se um número maior de eventos estressores no grupo institucionalizado, embora o impacto maior dos eventos tenha sido no grupo da família, o que demonstra o caráter subjetivo do que é considerado risco. Assim, para os participantes do grupo da família, os eventos são mais estressantes do que para o grupo institucionalizado. Isso pode sugerir que a vivência de um maior número de eventos fez com que os jovens abrigados percebessem alguns desses como menos impactantes do que os que moram com suas famílias. Infelizmente, o IEEIA não permite tal inferência, pois não contempla aspectos de estratégias de coping utilizadas sobre o evento, por exemplo. Trata-se de um ponto a ser mais investigado, para diferenciar casos adaptativos de ocorrências de desesperança e estagnação frente à adversidade.

Em relação ao impacto dos eventos, a diferença entre meninos e meninas novamente apareceu conforme o estereótipo reforçado socialmente (Steinberg ${ }^{9}$ ) e como em Kristensen et al. ${ }^{35}$ As meninas abrigadas tiveram médias mais altas de impacto em seis dos oito eventos, com diferença significativa em relação ao grupo das que moravam com sua família.

Sobre a freqüência de eventos por faixa etária, os resultados deste estudo corroboraram pesquisas anteriores que indicaram maior número de eventos negativos entre os adolescentes do que nas crianças nos dois contextos ${ }^{9,35}$. Esse resultado pode ser devido tanto ao acúmulo de eventos ao longo dos anos como ao aumento da demanda interna e externa durante a adolescência, em função do próprio desenvolvimento ${ }^{9}$. Também se torna importante enfatizar que o impacto atribuído aos eventos foi significativamente maior nos adolescentes somente em dois dos 60 itens ("não receber cuidado e atenção dos pais" e "ter dificuldades de ajustamento na escola”). Assim, como afirmaram Kristensen et $\mathrm{al}^{35}$, "se a análise ficasse limitada à freqüência dos eventos, poderia induzir à interpretação de que os níveis de estresse aumentam durante a adolescência: o que não parece ser o caso” (p. 50). Dessa forma, embora aumente o número de eventos, não há percepção aumentada do nível de estresse (impacto) quanto aos eventos vivenciados para esses jovens.

\section{Considerações finais}

O estudo da manifestação de sintomas depressivos em crianças e adolescentes pode ser uma importante ferramenta para melhorar a qualidade de vida das crianças brasileiras. Trata-se de um tema de saúde pública, visto que essa sintomatologia está associada a vários problemas de saúde mental. Este estudo demonstrou a associação entre a ocorrência de eventos estressores e a manifestação de sintomas depressivos entre crianças e adolescentes. Além disso, evidenciou quantitativamente que as crianças que estão afastadas de suas famílias biológicas, por medidas de proteção, apresentaram maiores escores de sintomas depressivos. Uma avaliação mais pormenorizada e longitudinal dos casos que ultrapassaram o ponto de corte de 24 pontos 
adotado poderia auxiliar na elaboração de um modelo explicativo dos fatores que influenciam essa situação.

Os eventos mais freqüentes apontados nesta amostra remetem aos microeventos diários como fontes de estresse, muitas vezes crônicos. Em linhas gerais, eventos como discutir com amigos e obedecer às ordens dos pais estão entre os resultados já obtidos em estudos anteriores $^{5,35,34,49}$. Da mesma forma, os eventos percebidos como mais impactantes foram eventos agudos e não esperados, como morte dos pais, o que também pode ser encontrado na literatura ${ }^{5,9,35,34,49}$. Os jovens abrigados apresentaram maior número de eventos estressores, pois, em geral, já sofreram algum tipo de negligência ou violência, passando pelo Conselho Tutelar, Juizado da Infância e Juventude, além de múltiplas avaliações profissionais. Essa trajetória provavelmente também contribuiu para atribuições de menor impacto aos eventos estressantes do que os jovens que moram com suas famílias.

Dentre as limitações deste estudo, seria importante a realização de uma investigação clínica, que permitisse uma avaliação mais consistente da manifestação dos sintomas depressivos nestes participantes, uma vez que o CDI não possibilita a obtenção de hipóteses diagnósticas. Além disso, seria importante também uma avaliação das estratégias de coping utilizadas em cada grupo, especialmente daqueles que obtiveram média baixa no CDI e número alto de eventos, para possibilitar programas de intervenção psicossocial, por exemplo. Outra limitação do estudo se refere ao seu caráter transversal, já que a avaliação foi realizada em apenas um momento, o que poderia refletir uma influência de circunstâncias desfavoráveis atuais. Assim, sugere-se a realização de estudos longitudinais, que também considerem a freqüência e a percepção do impacto do evento pela criança ou o adolescente e a manifestação dos sintomas depressivos ao longo do tempo.

Em suma, o presente trabalho contribuiu para destacar a necessidade de atenção aos jovens abrigados, tendo em vista os altos percentuais observados na ocorrência de eventos estressores e de sintomatologia depressiva. Além disso, sugere-se a discussão de políticas de atendimento a essa população, que observem o bem-estar psicológico de crianças e adolescentes em medidas de proteção.

\section{Referências}

1. Bahls SC. Aspectos clínicos da depressão em crianças e adolescentes. J Pediatr. 2002;78:359-66.

2. Bahls SC. Depressão: uma breve revisão dos fundamentos biológicos e cognitivos. Interação. 1999;3:49-60.

3. Dell’Aglio DD, Hutz CS. Depressão e desempenho escolar em crianças e adolescentes institucionalizados. Psicol Reflex Crit. 2004;17:351-7.

4. Dell'Aglio DD, Borges JL, Santos SS. Eventos estressores e depressão em adolescentes do sexo feminino. Psico. 2004;35(1):43-50.

5. Feijó RB, Raupp APG, John AB. Eventos estressores de vida e sua relação com tentativas de suicídio em adolescentes. J Bras Psiquiatr. 1999;48(4):151-7.

6. Kim J, Cicchetti D. Longitudinal trajectories of self-system and depressive symptoms among maltreated and nonmaltreated children. Child Dev. 2006;77:624-39.

7. Sternberg KJ, Lamb ME, Guterman E, Abbott CB. Effects of early and later family violence on children's behavior problems and depression: a longitudinal, multi-informant perspective. Child Abuse Negl. 2006;30:283-306.

8. Del Porto JA. Conceito e diagnóstico. Rev Bras Psiquiatr. 1999;21:6-11.

9. Steinberg L. Adolescence. 5th ed. Boston: McGraw-Hill; 1999.

10. Kessler RC, Avenevoli S, Ries Merikangas K. Mood disorders in children and adolescents: an epidemiologic perspective. Biol Psychiatry. 2001;49:1002-14.

11. Cunha JA. Manual da versão em português das Escalas Beck. São Paulo: Casa do Psicólogo; 2001.

12. Kovacs M. Children's depression inventory: technical manual update. Toronto: Multi-Health Systems; 2003.

13. American Psychiatric Association. Diagnostic and statistical manual of mental disorders: DSM-III-R. 3rd ed., rev. Washington: APA; 1987.

14. American Psychiatric Association. Diagnostic and statistical manual of mental disorders: DSM-IV. 4th ed. Washington: APA; 1994.

15. American Psychiatric Association. Diagnostic and statistical manual of mental disorders: DSM-IV-TR. 4th ed. rev. Washington: APA; 2000.

16. Baptista CA, Golfeto JH. Prevalência de depressão em escolares de 7 a 14 anos. Rev Psiquiatr Clin. 2000;27(5). Acesso em: 2006 jul. Disponível em: http://www.hcnet.usp.br/ipq/revista/27(5)/artigos/ art253.htm.

17. Kaplan HI, Sadock BJ, Greeb JA. Transtornos do humor e suicídio. In: Kaplan HI, Sadock BJ, Greeb JA, editors. Compêndio de psiquiatria: ciências do comportamento e psiquiatria clínica. $7^{\mathrm{a}}$ ed. Porto Alegre: Artmed; 1997. p. 1039-45.

18. Lima MS. Epidemiologia e impacto social. Rev Bras Psiquiatr. 1999;21:1-5.

19. Reppold CT, Hutz CS. Prevalência de indicadores de depressão entre adolescentes no Rio Grande do Sul. Aval. Psicol. 2003;2(2):175-84.

20. Siqueira AC, Betts MK, Dell'Aglio DD. A rede de apoio social e afetivo de adolescentes institucionalizados no sul do Brasil. R Interam Psicol. 2006;40(2):149-58.

21. Day VP, Telles LE de B, Zoratto PH, Azambuja MRF, Machado DA, Silveira MB, et al. Domestic violence and its manifestations. Rev Psiquiatr RS. 2003;25:9-21.

22. Garcia NM, Yunes MAM. Resiliência familiar: baixa renda e monoparentalidade. In: Dell’Aglio DD, Koller SH, Yunes MAM, editors. Resiliência e psicologia positiva: interfaces do risco à proteção. São Paulo: Casa do Psicólogo. 2006. p. 117-40.

23. Yunes MAM. Psicologia positiva e resiliência: o foco no indivíduo e na família. Psicol Estud. 2003;8:75-84.

24. Cowan PA, Cowan PC, Schulz MS. Thinking about risk and resilience in families. In: Hetherington EM, Bleachman EA, editors. Stress, coping and resiliency in children and families. Nova Jersey: Lawrence Erlbaum; 1996. p. 1-38.

25. Masten AS, Garmezy N. Risk, vulnerability and protective factors in developmental psychopathology. In: Lahey BB, Kadzin AE, editors. Advances in clinical child psychology. Nova York: Plenum; 1985. p. 1-52.

26. Heim C, Nemeroff CB. The role of childhood trauma in the neurobiology of mood and anxiety disorders: preclinical and clinical studies. Biol Psychiatry. 2001;49:1023-39.

27. Wong M. História e epidemiologia da depressão. In: Licinio J, Wong M, editors. Biologia da depressão. Porto Alegre: Artmed; 2007. p. 15-25.

28. McCloskey LA, Figueredo AJ, Koss MP. The effects of systemic family violence on children's mental health. Child Dev. 1995;66:1239-61. 
29. Pinheiro PS. Estudo das Nações Unidas sobre a violência contra crianças. Assembléia Geral das Nações Unidas. Acesso em: 2006 nov. Disponível em: http://www.unicef.org/brazil/pt/ Estudo_PSP_Portugues.pdf.

30. Brasil. Lei n. 8069, de 13 de julho de 1990. Estatuto da criança e do adolescente. Brasília, DF: Câmara dos Deputados; 1990.

31. Silva ERA, editor. O direito à convivência familiar e comunitária: os abrigos para crianças e adolescentes no Brasil. Brasília: IPEA/ CONANDA; 2004.

32. Porto Alegre, Prefeitura Municipal de Porto Alegre. Mapas e indicadores das vulnerabilidades sociais. Gerência de informações socioeconômicas, observatório da cidade de Porto Alegre. Acesso em: 2007 jul. Disponível em: http://lproweb.procempa.com.br/pmpa/ prefpoa/observatorio/usu_doc/mapas_e_indicadores_vulnerab _social_fasc_suas.pdf.

33. Gouveia V, Barbosa G, Almeida H, Gaião A. Inventário de Depressão Infantil - CDI: estudo de adaptação com escolares de João Pessoa. J Bras Psiquiatr. 1995;44:345-9.

34. Dell'Aglio DD. O processo de coping, institucionalização e eventos de vida em crianças e adolescentes [tese]. Porto Alegre: UFRGS; 2000.

35. Kristensen CH, Leon JS, D’Incao DB, Dell’Aglio DD. Análise da freqüência e do impacto de eventos estressores em uma amostra de adolescentes. Interação. 2004;8(1):45-5.

36. Conselho Nacional de Saúde. Resolução para pesquisas envolvendo seres humanos. Res. 196/1996. Brasília: CNS; 1997.

37. Conselho Federal de Psicologia. Resolução para pesquisas com seres humanos. Res. 016/2000. Brasília: CFP; 2000.

38. Bahls SC. Epidemiology of depressive symptoms in adolescents of a public school in Curitiba, Brazil. Rev Bras Psiquiatr. 2002;24:63-7.
39. Reppold CT. Estilo parental percebido e adaptação psicológica de adolescentes adotados [dissertação]. Porto Alegre: UFRGS; 2001.

40. Kovacs M. The Children's Depression Inventory (CDI). Psychopharmacol Bull. 1985;21:995-8.

41. Barbosa GA, Dias MR, Gaião AA, Lorenzo WCG. Depressão infantil: um estudo de prevalência com o CDI. Infanto Rev Neuropsiquiatr Infanc Adolesc. 1996;3:36-40.

42. Bailey MK, Zauszniewski JA, Heinzer MM, Hemstrom-Krainess AM. Patterns of depressive symptoms in children. J Child Adolesc Psychiatr Nurs. 2007;20:86-95.

43. Cole DA, Tram JM, Martin JM, Hoffman KB, Ruiz MD, Jacquez FM, et al. Individual differences in the emergence of depressive symptoms in children and adolescents: a longitudinal investigation of parent and child reports. J Abnorm Psychol. 2002;111:156-65.

44. Abreu SR. Transtornos psiquiátricos em crianças e adolescentes criados em instituições [dissertação]. São Paulo: UNIFESP; 2000.

45. Fitzpatrick KM. Exposure to violence and presence of depression among low-income African-American youth. J Consult Clin Psychol. 1993;61:528-31.

46. Carvalho AM. Crianças institucionalizadas e desenvolvimento: possibilidades e desafios. In: Lordelo ERA, Carvalho AMA, Koller SH, editors. Infância brasileira e contextos de desenvolvimento. São Paulo: Casa do Psicólogo; 2002. p. 19-44.

47. Siqueira AC, Dell’Aglio DD. O impacto da institucionalização na infância e na adolescência: uma revisão de literatura. Psicol Soc. 2006;18:71-80.

48. Rutter M. Resilience: some conceptual considerations. J Adolesc Health. 1993;14:626-31.

49. Dell'Aglio DD, Hutz SH. Eventos de vida de crianças e adolescentes institucionalizados. Aletheia. 2000;12:7-20. 